\title{
Avaliação do estado nutricional da mangueira Tommy Atkins no submédio do vale do rio São Francisco: cálculo dos índices DRIS
}

Recibido: 7 outubro 2008 / Aceptado: 11 decembro 2008

(C) IBADER- Universidade de Santiago de Compostela 2010
Resumo O Sistema Integrado de Diagnose e Recomendação, DRIS, é uma ferramenta importante na avaliação do estado nutricional das plantas. Os objetivos do presente trabalho foram avaliar o estado nutricional de pomares de mangueira Tommy Atkins no Submédio do vale do São Francisco, no Brasil, mediante o DRIS, relacionar os índices com os teores foliares de nutrientes e discriminar a ordem de limitação de cada nutriente nas subamostras de alta e de baixa produtividade. Foram amostrados sessenta e três pomares comerciais com sete ou mais anos de idade. Com os teores foliares de macro e micronutrientes foram calculados os índices DRIS, utilizando-se como referência os dados da subpopulação de alta produtividade. Os maiores valores de Índices de Balanço Nutricional médio $\left(\mathrm{IBN}_{\mathrm{m}}\right)$ verificados em pomares de baixa produtividade

\section{P. A. da C. Pinto}

Prof. Pleno, Departamento de Tecnologia e Ciências Sociais, DTCS da Universidade do Estado da Bahia, UNEB, Cx. Postal 171, 48905680 Juazeiro - BA, Fone: (74) 36117363.

http://www.pacostapinto.com.br

e-mail: pacostapinto@hotmail.com

\section{E. Dias}

Prof. do Depto. de Solos da UFV, 36571000 Viçosa, MG. ledias@ufv.br

V. H. Alvarez V.

Prof. do Depto. de Solos da UFV, 36571000 Viçosa, MG. vhav@ufv.br

M. M. Choudhury

Pesquisador científico do CPATSA/EMBRAPA, Cx. Postal 23, 56 302970 Petrolina - PE. mohammad@cpatsa.embrapa.br

G. Vieira

Prof. do Depto. de Fitotecnia da UFV, 36570000 Viçosa, MG. gerival@ufv.br demonstraram o potencial deste índice na avaliação do estado nutricional da mangueira, e a necessidade de adubações mais criteriosas em tais pomares. A utilização do conceito de potencial de resposta à adubação na interpretação dos índices DRIS mostrou-se uma ferramenta eficaz, principalmente nos pomares de baixa produtividade, onde os desequilíbrios nutricionais foram maiores, confirmando a seqüência de deficiência a excessos, auxiliando o diagnóstico final, indicando os nutrientes que realmente estão em deficiência ou em excesso.

Palabras chave Macronutrientes - micronutrientes, diagnose foliar · equilíbrio nutricional · adubação

Summary The Integrated System diagnosis and Recommendation, DRIS, is an important tool in assessing the nutritional status of the plants. The aims of this study were to assess the nutritional status of mango orchards of Tommy Atkins in Submédio of valley of São Francisco, in Brazil, through the DRIS, linking the rates with the levels of nutrients and leaf discriminate on the order of limitation of each nutrient sub samples of high and low productivity. Were sampled sixty-three commercial orchards with seven or more years old. With the leaf tenors of macro and micronutrients DRIS indexes were calculated, using as reference data from the subpopulation of high productivity. The highest average indices of Nutritional Balance $\left(\mathrm{IBN}_{\mathrm{m}}\right)$ recorded in orchards of low productivity demonstrated the potential of this index in assessing the nutritional status of the mango, and the need for fertilization more wise in such orchards. The use of the concept of potential response to fertilization in the interpretation of the indices DRIS proved to be an effective tool, particularly in low productivity of orchards, where the nutritional imbalances were higher, confirming the sequence of the excess to deficiency, helping the final diagnosis, indicating the nutrients what really are in deficiency or excess.

Keywords Macronutrient · micronutrient · diagnsis · balance nutritional · fertilización 


\section{Introdução}

É notável a capacidade de plantas converterem água, ar, luz solar e nutrientes em alimentos, fibras e outras substâncias indispensáveis ao homem mediante a fotossíntese, fazendo desses fatores um pré-requisito fundamental para a vida humana e animal sobre a terra (Bergmann, 1992). No entanto, essa capacidade de conversão é comprometida quando desequilíbrios nutricionais são estabelecidos.

Stassem et al. (1997), estudando a absorção e distribuição de macro e de micronutrientes em árvores de mangueira, cultivar Sensation enxertada sobre o cv. Sabre, com 2, 6 e 18 anos de idade, determinaram as quantidades de macronutrientes que se acumulam em diferentes partes da árvore (raízes, casca, lenho, ramos novos, folhas e frutos), concluindo que em árvores de seis anos de idade as folhas apresentam as maiores partições de $\mathrm{N}, \mathrm{P}, \mathrm{K}, \mathrm{Ca}$ e $\mathrm{Mg}$ em relação às demais partes estudadas. Isso também foi observado para as árvores de 2 e de 18 anos, com algumas exceções, a saber: $P$ (em raízes, casca e lenho de árvores de 2 anos e em ramos novos e frutos de árvores de 18 anos); K (em frutos de árvores de 2 e de 18 anos; em ramos novos de árvores de 18 anos); $M g$ (raízes de árvores de $2 \mathrm{e}$ de 18 anos; frutos de árvores de 2 anos), cujos teores foram maiores que nas folhas. Mais de $40 \%$ do Ca contido na árvore encontram-se nas folhas.

O Sistema Integrado de Diagnose e Recomendação, DRIS, é uma ferramenta importante na avaliação do estado nutricional das plantas. Para tanto selecionam-se pomares que constituam a subamostra de referência, da qual se obtém os teores foliares e produtividades respectivas. Esta subamostra é submetida a um teste de normalidade. O cálculo dos índices exige que as relações (quocientes) entre os teores dos nutrientes tenham sua origem numa subamostra em que os quocientes entre nutrientes sigam distribuições normais $h(m, s)$, de média $m$ e desvio padrão $\mathrm{s}$, ou melhor, uma distribuição normal reduzida $\mathrm{h}(0,1)$ (Alvarez V. \& Leite, 1999).

De acordo com Schaffer et al. (1988) o DRIS é um procedimento integrado que identifica a suficiência de cada nutriente em relação a outros na planta. O índice DRIS de um nutriente é calculado para cada nutriente. Quando o índice de um nutriente torna-se mais negativo, aumenta a probabilidade de que o mesmo limite o crescimento da planta e a sua produção. O somatório dos valores absolutos destes índices forma o Índice de Balanço Nutricional (IBN) (Baldock \& Schulte, 1996), que expressa o equilíbrio nutricional da lavoura amostrada. Quanto menor o IBN, menor será o desequilíbrio entre nutrientes (Snyder \& Kretschmer, 1988). O DRIS pode prover um procedimento útil para identificar desbalanços nutricionais e identificar os nutrientes mais limitantes associados com outros fatores bióticos, tais como doenças, que podem limitar a produção (Schaffer et al., 1988).

Os objetivos do presente trabalho foram avaliar o estado nutricional de pomares de mangueira Tommy Atkins no Submédio São Francisco, mediante o DRIS, relacionar os índices com os teores foliares de nutrientes e discriminar a ordem de limitação de cada nutriente nas subamostras de alta e de baixa produtividade.

\section{Material e Métodos}

Sessenta e três pomares de mangueira Tommy Atkins em plantios comerciais com sete ou mais anos de idade, localizados no Nordeste do Brasil, foram amostrados no período de 1997 a 1999. Escolheu-se em cada pomar um talhão com árvores com sete ou mais anos de idade, porte uniforme e estado sanitário adequado. Amostras foliares foram obtidas em cada ano, na porção mediana da copa, nos quatro pontos cardeais (Quaggio, 1996), no penúltimo lançamento dos ramos, em vinte e cinco árvores do talhão, na semana anterior à das pulverizações das copas com nitratos, objetivando a quebra da dormência das gemas terminais. As folhas, depois de lavadas em água destilada, foram colocadas em estufa com circulação mecânica de ar, à temperatura de 65 a $70^{\circ} \mathrm{C}$ até peso constante, após o que foram moídas em moinho Wiley e passadas em peneira de $1 \mathrm{~mm}$ de malha.

$\mathrm{O} \mathrm{N}$ foi determinado após digestão sulfúrica, pelo método micro Kjeldahl. Os elementos P, K, Ca, Mg, Cu, Fe, Mn e Zn foram determinados após digestão nítrico-perclórica. O B foi determinado, após incineração da amostra em forno elétrico a $550{ }^{\circ} \mathrm{C}$, por colorimetria de curcumina (Malavolta et al.,1997). O $\mathrm{P}$ foi determinado por espectrofotometria com azul-de-molibdênio. O Ca, o $\mathrm{Mg}$, o $\mathrm{Cu}$, o $\mathrm{Fe}$, o $\mathrm{Mn}$ e o $\mathrm{Zn}$ foram determinados empregando-se espectrofotômetro de absorção atômica. O K foi determinado por fotometria de chama (Malavolta et al.,1997).

Com os teores foliares de N, P, K, Ca, Mg, B, Cu, Fe, Mn e $\mathrm{Zn}$ foram calculados os índices DRIS, utilizando-se como referência os dados da subpopulação de alta produtividade, que foram processados pelo software Floresta 1.0 (Wadt, 1995). A fórmula de cálculo dos índices DRIS utilizada foi a proposta por Jones (1981), equivalente à de Beaufils (1973), na qual os desvios negativos não são superestimados.

No cálculo das funções $Z$ (A/B), utilizou-se a fórmula recomendada por Jones (1981), ou seja: $Z(A / B)=(A / B-$ $a / b) k / S$, onde $Z(A / B)$ representa a função da relação entre os nutrientes $A$ e $B$ do pomar sob diagnóstico; $A / B$ representa o valor da relação entre nutrientes $A$ e $B$, para o pomar a ser diagnosticado; a/b representa o valor da média obtida para as relações oriundas da subamostra de plantas de alta produtividade (norma); $\mathrm{k}$ é um coeficientes, neste trabalho igual a 1,0; $S$ é o desvio padrão dos valores da relação a/b na subamostra de referência.

$\mathrm{O}$ índice de Balanço Nutricional médio $\left(\mathrm{IBN}_{\mathrm{m}}\right)$ foi calculado mediante o somatório dos valores absolutos dos índices DRIS (Leite, 1993; Costa, 1995), obtidos para cada nutriente em cada pomar, dividido pelo número de nutrientes considerados $(n)$, conforme a equação:

$$
\left|\mathrm{BN}_{m}=\right| \text { indice } \mathrm{A}|+| \text { incices } \mathrm{B}|+\ldots+| \text { indices } \mathrm{N} \text { in }
$$


O cálculo dos índices para cada nutriente foi realizado pelo software Floresta 1.0 (WADT, 1996) empregando-se a fórmula descrita por Beaufils (1973), onde se utiliza a média das relações diretas e inversas e onde $\mathrm{N}$ corresponde ao número de nutrientes envolvidos, ou seja:

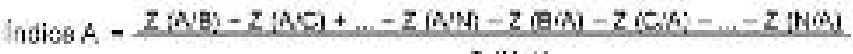
$2\langle\mathrm{~N}-1\rangle$

Para a interpretação dos índices DRIS (Tabela 1), incorporou-se o conceito de potencial de resposta à adubação (PRA) (Wadt, 1996). Para tanto, nos pomares de baixa e de alta produtividade, os nutrientes foram classificados segundo o potencial de resposta à adubação em respostas: positiva (p), positiva ou nula (pz), nula (z), negativa ou nula (nz) e negativa $(n)$. Posteriormente, visando maior síntese, foi feito o agrupamento das duas primeiras e das duas últimas classes de resposta, nas classes de status nutricional: limitante por falta (LF), limitante por excesso (LE) e não limitante (NL), esta última representada pela resposta nula à adubação.

\begin{tabular}{|c|c|c|c|c|}
\hline Irusice: & Misduso do indice & $+\mathrm{LF} a u+L E$ & $\begin{array}{c}\text { Potencial do resposta à } \\
\text { adubuçis }\end{array}$ & $\begin{array}{c}\text { Clasese de status } \\
\text { nutricional }\end{array}$ \\
\hline$<0$ & $>1 B W_{h}$ & $\sin$ & F & LF \\
\hline$<0$ & $>1 B N_{1}$ & กลี่อ & $p^{2}$ & IF \\
\hline$>0$ & $=18 \mathrm{~N}$ & $\sin$ & ก & LE \\
\hline$>0$ & $>I B N_{1}$ & nde & $n z$ & LE \\
\hline$\leq 0$ & $\leq I B N_{n}$ & indepordente & $z$ & NL \\
\hline$\geq 0$ & $\leq I B N$, & independente & $z$ & NL \\
\hline
\end{tabular}

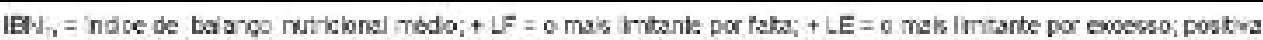

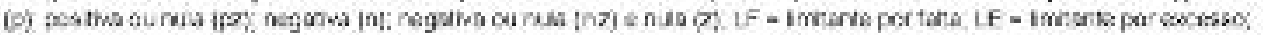
P.L $=$ rso Imtarts

\section{Resultados e Discussão}

Nas Tabelas 2 e 3 são mostrados os índices DRIS calculados para os nutrientes N, P, K, Ca, Mg, B, Cu, Fe, Mn e $\mathrm{Zn}$, os Índices de balanço Nutricional (IBN), a classe de solo e a ordem de limitação de nutrientes nos pomares de mangueira Tommy Atkins de baixa e de alta produtividade no Submédio São Francisco, Nordeste do Brasil.

$\mathrm{Na}$ Tabela 4 estão as equações e os coeficientes de correlação que descrevem as relações entre índices DRIS e os teores foliares $(x)$ de macro e de micronutrientes contidos em árvores da população total estudada. Constata-se haver uma correlação positiva entre os teores foliares de nutrientes e os índices DRIS, donde se depreende que tais índices são úteis ao se efetuar a diagnose nutricional.

A menor correlação constatada entre os teores foliares de $\mathrm{N}$ e os correspondentes índices DRIS, pode estar associada ao manejo do elemento nos pomares, uma vez que podem existir variações quanto a quantidade, tipo de fertilizante e épocas de adubação; da mesma forma, o K, considerado o elemento mais exportado pela cultura, sendo também aplicado via foliar por ocasião do pré-florescimento. Portanto, a menor correlação observada para esse nutriente, igualmente pode ser explicada em função de uma falta de padrão entre os agricultores quanto ao manejo da adubação potássica.

Na Tabela 5 são apresentadas as porcentagens em que os macro e micronutrientes ocorrem na ordem de limitação por deficiência nutricional nos pomares de baixa e de alta produtividade estudados na Região do Submédio São Francisco.

Em 52,73 \% dos pomares de alta produtividade os macronutrientes são os mais limitantes por deficiência, destacando-se $\mathrm{Mg}, \mathrm{K}$ e Ca, enquanto que em 46,87 \% dos pomares os micronutrientes foram os mais limitantes por deficiência, destacando-se Fe e Zn (Tabela 5). Maior atenção precisa ser dada à análise de micronutrientes (no solo e na planta), com conseqüente aplicação desses nos programas de adubação da cultura.

Em 36,24 \% dos pomares de baixa produtividade os macronutrientes são os mais limitantes por deficiência, destacando-se o $\mathrm{Ca}$, enquanto que em 63,04 \% desses pomares os micronutrientes foram os mais limitantes por deficiência, destacando-se B, Cu e Zn (Tabela 5).

Na subpopulação de alta produtividade ( $\geq 250 \mathrm{~kg} /$ planta) os teores foliares de Ca e de Mn, são da ordem de 15,0 a 48,0 $\mathrm{g} / \mathrm{kg}$ e de 120,0 a $1.150,0 \mathrm{mg} / \mathrm{kg}$, respectivamente, tendo-se constatado uma correlação negativa entre os teores dos mesmos. Em alguns pomares aparentemente bem conduzidos, a despeito das adições de cálcio ao solo, temse ainda a ocorrência de distúrbios fisiológicos nos frutos, como o colapso interno, em níveis da ordem de 16 a $17 \%$, com relações $\mathrm{Ca} / \mathrm{N}$ foliares entre 0,75 e 2,64 (enquanto a norma $=1,59$ ) e relação $\mathrm{Ca} / \mathrm{Mn}$ entre 0,018 e 0,067 (norma $=0,04)$ (Pinto, 2002), sugerindo que outros fatores, além da relação $\mathrm{Ca} / \mathrm{N}$, contribuem para a manifestação de tal problema que deprecia o fruto, inviabilizando a sua comercialização e consumo.

$\mathrm{Na}$ indução de sintomas de deficiência de $\mathrm{Ca}$ (folha dobrada) em tecidos com alto teor de Mn é muito provável haver um efeito indireto sobre o transporte de $\mathrm{Ca}$ para folhas em expansão. O transporte de Ca é mediado por um contra-transporte de ácido indol acético (AIA), e alta atividade de AIA oxidase, ou em geral pela atividade de polifenoloxidase, o que é uma característica típica em tecidos com alto teor de $\mathrm{Mn}$. Sintomas de deficiência de $\mathrm{Ca}$ 
induzida por toxicidade de Mn são provavelmente causados por elevada degradação de AIA. Um processo agravado, por exemplo, por alta intensidade de luz (Horst, 1988).

A faixa de teor de $\mathrm{Mn}$ interpretada como adequada na região (Quaggio, 1996) é da ordem de 50 a $100 \mathrm{mg} / \mathrm{kg}$, enquanto que nos pomares estudados neste trabalho a faixa de variação encontrada foi da ordem de 120 a 1.500 $\mathrm{mg} / \mathrm{kg}$, encontrando-se pomares com 293, 360, 750, 1.000 e $1.150 \mathrm{mg} / \mathrm{kg} \mathrm{Mn}$, produzindo 500, 437, 297, 423 e 377 $\mathrm{kg} /$ planta, respectivamente, o que são produtividades excelentes, não sendo, aparentemente, esses altos teores prejudiciais à cultura. Esses altos teores foliares de $\mathrm{Mn}$ podem ser decorrentes de pulverizações de produtos fungicidas como mancozeb (etileno bis - ditiocarbonato de $\mathrm{Zn}$ e $\mathrm{Mn}$ ) que poderia estar se acumulando nas folhas.

A luz afeta a absorção de Mn pela planta, estimulando-a (Hewitt, 1963, citado por Camargo \& Silva, 1990). Também esse fato pode ser agregado à explicação dos elevados teores de $\mathrm{Mn}$ nas folhas da mangueira Tommy Atkins na região do Submédio São Francisco, onde se tem por ano cerca de 3.000 horas de luminosidade solar.

Conforme Samra \& Arora (1997) a concentração foliar de Fe e de $\mathrm{Mn}$ em folhas de mangueiras foi fotossensível e dependente da direção ou lado da árvore em relação ao movimento do sol.

\begin{tabular}{|c|c|c|c|c|c|c|c|c|c|c|c|c|c|}
\hline POMKR & Ordem & $\mathbf{N}$ & $P$ & $\mathbf{K}$ & $\mathrm{Ca}$ & hes & $\mathrm{B}$ & $\mathrm{Zn}$ & Mn & Fe & Cu & IBN. & ORDEM DE LMITACAAO \DEFICIENCIA A E \\
\hline 1 & $x+a$ : & 00 & 0,60 & 50) & $\infty 0$ & 40 & 2 & 57 & 22 & $+\infty$ & 053 & 0.76 & $9=\mathrm{N}=\mathrm{O}=\mathrm{K}=\mathrm{Ha}$ \\
\hline 2 & Argescto & 0,20 & 1,02 & 0,13 & $-0,12$ & $-0,75$ & $\rightarrow \$ M$ & 0,52 & $-0,52$ & 138 & $0 \leq 2$ & 670 & $\theta=M g>M n=N>C a>K>Z r>C u>P>F e$ \\
\hline$y$ & $\cdot$ & a, 6 & 0,47 & $\theta 0$ & $-0,00$ & -o:3s & -0.50 & 0,45 & Q 16 & Q & 0.44 & $\mathrm{Q}<\mathrm{i}$ & $s+C a>\theta>M J>K>M>P>E u>Z n>F e$ \\
\hline 4 & Ngrsolo & 0,55 & 2,20 & $0.9 ?$ & 0,29 & 077 & $-4,14$ & 0,78 & 2,69 & 1,00 & 0,35 & $\square 32$ & $\exists>M g>A_{n}>M=C u>k>C a>Z_{n}>F \in>P$ \\
\hline 5 & Angsedo & 0,84 & 0.71 & 095 & -0.10 & -010 & $-1,02$ & $-1,70$ & $-0,85$ & 4,74 & 322 & 113 & $\mathrm{Zn}>\mathrm{e}>\mathrm{Mn}>\mathrm{N}>\mathrm{K}>\mathrm{Cal}>\mathrm{Kg}>\mathrm{F}>\mathrm{Fe}>\mathrm{Cu}$ \\
\hline 6 & Argasols & 0,16 & $1,4 \bar{r}$ & 0,44 & $-0,12$ & Don & $-1,23$ & $-\pi, \Phi$ & $-2,05$ & 222 & $-1,136$ & 1074 & $\mathrm{Eu}>\mathrm{B}>\mathrm{K}>\mathrm{N}>\mathrm{Ca}>\mathrm{Zn}>\mathrm{Mn}>\mathrm{Ng}$ \\
\hline 7 & Angrack & 0.30 & $2 x$ & 0.37 & 0.44 & 109 & Q.a1 & $-7, a x$ & +91 & $\mathrm{a}<\mathrm{a}$ & $-1,80$ & $1 \leq 6$ & $7 n>C u>K>N>B>C a>N_{B}>V_{n}>F>F n$ \\
\hline 8 & A givact: & 2,22 & 3.90 & 2.49 & 225 & 172 & $-16,14$ & $-1,07$ & 7.68 & 424 & -1.41 & 272 & $\mathrm{~A}=\mathrm{Cu}+\mathrm{Zn}_{n}>\mathrm{N}_{1}>\mathrm{K}_{\mathrm{g}}+\mathrm{C}_{2}>\mathrm{N}>\mathrm{K}>$ \\
\hline 9 & Angsion & Q38 & 042 & 0.70 & $-1 \mathrm{ca}$ & -0.25 & -1.13 & 0,25 & 011 & $+7 \mathrm{~F}$ & Q*1 & 0.56 & $\mathrm{~B}=\mathrm{C}=\mathrm{N}>\mathrm{Mg}>\mathrm{K}>\mathrm{Mn}>\mathrm{S}=\mathrm{Zn}>\mathrm{P}=\mathrm{Fe}$ \\
\hline 19 & Miguesch & QAS & 1,08 & $\Leftrightarrow \$$ & 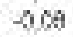 & $0 \leqslant 9$ & -0.70 & -1.11 & $\Delta \in 2$ & -1.01 & 1,19 & 889 & $B=k>C h+h$ \\
\hline 75 & Lexcesedo & $a, 23$ & 6,80 & $\Leftrightarrow, 18$ & $-0,44$ & $0 \leqslant 5$ & -0.98 & 7,17 & 0,58 & 206 & $-3,24$ & 1,00 & $\mathrm{Cu}>\mathrm{B}>\mathrm{C} a>\mathrm{N}>\mathrm{K}>\mathrm{Ng}>\mathrm{N}>\mathrm{P}>\mathrm{Zn}>\mathrm{Fe}$ \\
\hline 15 & Lunseata & 045 & 079 & $\rightarrow$ Aा & $a x$ & $0 \geqslant 7$ & $-1,4$ & -1.28 & a. 18 & 7,73 & Q97 & $8 \geqslant 1$ & 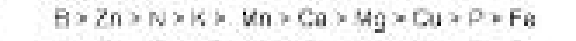 \\
\hline 98 & Angenoto: & 0.35 & 1,10 & 129 & $-0,23$ & 0 ob & $-0,11$ & $-0,6 n$ & $0 \leqslant 0$ & 5,6 & $-0, \pi$ & 4,2 & $K>\mathrm{Cu}>\mathrm{Zn}>\mathrm{Cu}>\mathrm{Nu}>\mathrm{B}>\mathrm{Nr}>\mathrm{Kg}>\mathrm{P}>\mathrm{Fe}$ \\
\hline 20 & o & 0,27 & 0,58 & 0.15 & 0,80 & 018 & 4,48 & 0,30 & व.81 & 206 & $-1,4$ & $\square 82$ & $J>B>C s>Z r>N>K>N g=$ \\
\hline 22 & $\cdot$ & a, & 0,85 & 0,28 & 0,02 & $\mathrm{v} z 2$ & 3,03 & 0,57 & $-2,65$ & $\pi, 05$ & $-6,28$ & 1,17 & $\mathrm{Cu}>\mathrm{M} n>\mathrm{Cs}>-\mathrm{e}>\mathrm{Mg}>\mathrm{K}>\mathrm{Zn}>\mathrm{N}>$ \\
\hline 24 & Wertissab. & Q93 & 242 & 0.04 & $1 \pi$ & $-1,13$ & 0.41 & $-4,22$ & 322 & 230 & $-1,16$ & 115 & $>Q=N \theta>N=Z n>K>B>M n$ \\
\hline 28 & Angisock & $-1,02$ & 0.2 & 042 & -0.19 & $0: 1$ & 0.65 & $-0,28$ & $a<9$ & 7,20 & -1.17 & Q 61 & $\mathrm{Cu}>\mathrm{M}>\mathrm{K}>\mathrm{Zn}>\mathrm{Ca}>\mathrm{Mg}>\mathrm{Mh}>\mathrm{P}>\mathrm{E}>\mathrm{Fe}$ \\
\hline 27 & Angescols & a.s & 0,49 & 012 & ㅁ. 13 & 032 & $-1,22$ & $-4,2$ & $\mathbb{1 2}, 2$ & 1,25 & $-0,2$ & $\mathrm{D}<\mathrm{i}$ & $B>P>O \mu Z n>K>N>C a$ \\
\hline 30 & cos & 0,51 & 097 & 0.73 & -0.40 & 1.12 & 0,16 & $-0,80$ & $-0,19$ & +11 & $-0,98$ & $\triangle 60$ & $\mu>Z n>N>C s>M n>B>P>K$ \\
\hline 34 & Lebeasds & 0,08 & 0.44 & $0 x$ & -0.03 & -0.48 & $-2,17$ & $-0,70$ & 291 & $.0,28$ & 0,25 & L84 & $\mathrm{Zn}>\mathrm{M}_{3}>\mathrm{F}_{u}=\mathrm{B}>\mathrm{N}>\mathrm{Cb}>\mathrm{K}>\mathrm{Cu}$ \\
\hline 38 & $\cdot$ & 2,45 & 0.3 & $0, x$ & 03 & 0.29 & $-0,28$ & $-1,78$ & I.14 & A 31 & 1.25 & I] $\Delta \mathrm{fi}$ & $2 n>N>H_{g}>3>K>P>M n=\mathrm{Aa}>$ \\
\hline 90 & $\mathrm{n}$ & Q:1 & $-1 / 78$ & & & a.s. & & $-2,18$ & & $9 \cdot 9$ & $1,1: 7$ & & \\
\hline$\$ 0$ & + & 2,89 & 0,11 & 075 & 0,70 & -0.12 & -0.43 & 0,51 & 020 & $-2,09$ & 1,65 & i & $\Rightarrow \mathrm{Ca}>\mathrm{B}>\mathrm{Hg}>\mathrm{P}>\mathrm{Mr}$ \\
\hline 41 & + & 0,82 & 0,08 & $0, \infty$ & $0, \tau$ & $0 \geq 2$ & 0,94 & 0,69 & & & & & - \\
\hline 42 & oesole & $a, 0$ & $0 \pi$ & 000 & $-1,30$ & 000 & 0,20 & 5,01 & $-0,21$ & 121 & 2.25 & dis & $: K>P=N>M n>M O B$ \\
\hline 43 & & 0,70 & 040 & $0 x$ & 0,78 & 086 & $-5,50$ & 0,45 & $\alpha 32$ & 1,02 & 0.57 & $\pi G$ & $P=K=2$ \\
\hline 44 & solo & 0,35 & 0,24 & 0,97 & $-0,64$ & 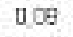 & $-0,83$ & 0,83 & & & $-0,98$ & 048 & $\mathrm{Mn}_{n}>\mathrm{Fe}>\mathrm{MQ}$ \\
\hline 48 & Angssodo & 0,46 & 027 & 0,0 & $0 \propto 2$ & 219 & $-4,61$ & 0,52 & -2. & -2 & $2 E$ & 105 & $H_{a}=\mathrm{C}$ \\
\hline 47 & & 0,4 & $0 \%$ & $-0,53$ & $-0,49$ & -0.63 & $-4,30$ & 0,53 & & & 6.89 & 1.49 & $\mathrm{Cu}$ \\
\hline 45 & Anglacek. & 0,50 & -197 & -032 & -0.24 & -122 & $-0,11$ & $-1, \pi 5$ & 270 & $.2,97$ & 6,00 & 134 & $Z_{n}>\mathrm{Ma}_{3}>\mathrm{P}>\mathrm{F} a>\mathrm{Ca}>\mathrm{N}>\mathrm{K}>\mathrm{B}>\mathrm{U}_{n}>\mathrm{Cu}$ \\
\hline 49 & Nocasobs: & -1.75 & 103 & $0, \%$ & $-0,29$ & $-0,4$ & 9,28 & 1,50 & -2.70 & $80 \mathrm{D}$ & $-1 \times 0$ & D. 84 & 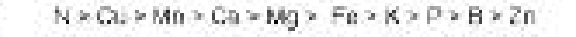 \\
\hline 50 & alt; & ans & 0,19 & $O S$ & $-0,12$ & 0.11 & -0.78 & -0.71 & 1,10 & 0.78 & 0.97 & $0<9$ & $\mathrm{Cu}=\mathrm{B}=\mathrm{Zn} \times \mathrm{C}_{\mathrm{d}} \times \mathrm{N}>\mathrm{V}_{\mathrm{i}} \times \mathrm{P} \times \mathrm{K}$ \\
\hline 51 & $\sec b$ & 0.28 & $0,7 ?$ & 0,20 & $-0,80$ & 0.15 & 0,85 & 0,09 & $-1,52$ & 089 & 038 & 050 & $\mathrm{Hn}>\mathrm{Ca}>\mathrm{Zn}>\mathrm{Mg}>\mathrm{P}>\mathrm{K}>\mathrm{N}>\mathrm{O}_{4}>\mathrm{Fe}>\mathrm{B}$ \\
\hline 55 & asicte & $a \cdot 5$ & 0,72 & 0,76 & 0.12 & 014 & 0,18 & $-6,07$ & $-1,51$ & 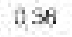 & a.4 & {$[130$} & $1 \times 2 n+B>$ \\
\hline 54 & Lativesola & a,e1 & $\oplus \sqrt{7}$ & $0 * 2$ & $-0,01$ & 014 & 1,00 & -7.10 & 7,14 & $A D Q$ & $-1,76$ & & $>2 \mathrm{n}>\mathrm{N}>\mathrm{P}>\mathrm{Cr}>\mathrm{Fe}>\mathrm{Mu}>\mathrm{A}$ \\
\hline 55 & $\cdot$ & 0,05 & 0,90 & 0,20 & 0,28 & -062 & 0,95 & $-0,0$ & $-2,78$ & $\cdot 0$ & $-1,21$ & $0<0$ & $\mathrm{Cu}_{u}>\mathrm{M}_{2}>\mathrm{M}_{7}>\mathrm{F}_{0}>\mathrm{Zn}>\mathrm{N}>\mathrm{K}>\mathrm{C}>\mathrm{B}>\mathrm{P}$ \\
\hline SE & $\cdot$ & ans & 1,31 & 0,05 & $-0,201$ & 03 & -0.42 & $-0,12$ & $\infty, 13$ & & $-0,14$ & $0: 4$ & $\mathrm{Fe}_{0}>\mathrm{Ca}>\mathrm{B}>\mathrm{Un}_{\mathrm{n}}>\mathrm{Cu}>\mathrm{Zn}>\mathrm{N}>\mathrm{Mg}_{\mathrm{g}}>\alpha>\mathrm{P}$ \\
\hline 57 & Angseods & 0,05 & $-1,32$ & 0,07 & 2,64 & 0.64 & 0,30 & 2,51 & व 10 & 1 & $5, \mathrm{e} 1$ & 130 & $\mathrm{Zn}>\mathrm{Fe}_{\mathrm{e}}>\mathrm{P}=\mathrm{Ca}=\mathrm{Ug}_{\mathrm{g}}>\mathrm{g}>\mathrm{N}>\mathrm{K}=\mathrm{Un}>\mathrm{Cu}$ \\
\hline 55 & $\cdot$ & 1,15 & 0,07 & 0,20 & $0 z 8$ & vor & 0,44 & $-3,69$ & 2,14 & 126 & 1,00 & 0,72 & $2 n>P>M g>N n>\langle>-e>C s>B>C u>N$ \\
\hline E0 & knossabs. & 0.35 & 2 at & $0 \pi$ & $\mathrm{n} 34$ & 074 & $-0,27$ & $-3, \%$ & $-2,69$ & $a<1$ & QXa & $10 \theta$ & $Z n>M n>N>a>C a>F e>C u>K>M g>P$ \\
\hline 92 & $\cdot$ & 0,03 & 0,08 & 0.45 & $0: 2$ & 000 & $-1,19$ & 0,42 & $-2,63$ & $-2,10$ & 0.23 & 036 & $\exists>\mathrm{Hn}>\mathrm{FD}>\mathrm{Vg}>\mathrm{N}>\mathrm{C}>\mathrm{Cu}>\mathrm{Z}_{n}>\mathrm{K}>\mathrm{F}$ \\
\hline 53 & * & 0,25 & 034 & 012 & -0.43 & 027 & $-0,0$ & 9,31 & $-0,64$ & $\square 38$ & $.0 \pm$ & 428 & $a>M n>C u>C a>N>K>M g>P>T_{e}>Z n$ \\
\hline 54 & $\rightarrow$ & Q390 & 1,15 & 192 & $-0,42$ & 0 st & $-6,28$ & 7.32 & $-2,08$ & $2 \pi$ & $-0,2$ & $15 \mathrm{SA}$ & 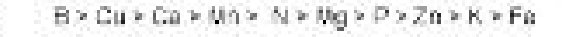 \\
\hline SB & A & 0.4 & 042 & 0.39 & $-1,08$ & $0 \leqslant 8$ & +1.48 & 1,07 & $-2,43$ & 0.38 & 9.65 & 0.59 & 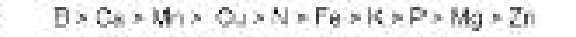 \\
\hline 87 & + & 0,89 & $+2,05$ & 0,32 & $-1,70$ & 0.78 & $\because, 2$ & $-0,28$ & 0,61 & 7,06 & acs & 0,8 & $\mathrm{Cs}>\mathrm{B}>2 \mathrm{n}>\mathrm{P}>\mathrm{Cu}>\mathrm{K}>\mathrm{Mn}>\mathrm{N}>\mathrm{Mg}>\mathrm{Fe}$ \\
\hline 05 & $\cdot$ & a.s & $-1 \leq 2$ & $-12 i$ & $-1,00$ & non & -2.17 & 2,17 & $42 i$ & 400 & 2.5 & 111 & 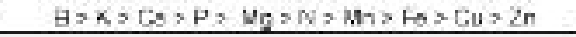 \\
\hline
\end{tabular}

Tabela 2.- Ordens de Solo, Índices de balanço Nutricional médio (IBNm), Índices DRIS e Ordem de Limitação de nutrientes obtidas a partir de teores foliares de nutrientes em pomares de mangueira Tommy Atkins de baixa produtividade $(<250 \mathrm{~kg} /$ planta) no Submédio São Francisco 


\begin{tabular}{|c|c|c|c|c|c|c|c|c|c|c|c|c|c|}
\hline OMAR & Ordem & N & $\mathbf{P}$ & $\kappa$ & Co & $\mathrm{my}$ & B & $Z_{n}$ & Min & $\mathrm{Fe}$ & $\mathrm{Ou}$ & I8N. & ORDERA DE LIIITAÇAO \{DEFICIÉNCLA A EXCES9O\}) \\
\hline 10 & Arginests & $-\pi, s$ & 6,20 & $-1,21$ & $-0,42$ & $-0,75$ & $-11<2$ & 1,30 & $-0,111$ & 023 & 0.56 & 958 & $\mathrm{Cg}>\mathrm{M}_{\mathrm{I}}>\mathrm{B}>\mathrm{N}>\mathrm{K}>\mathrm{Mn}>\mathrm{Fo}>\mathrm{P}>\mathrm{Cu}>\mathrm{Zn}$ \\
\hline 12 & Arganet: & ax $x$ & 011 & 0,65 & -0.51 & 0.78 & 641 & -1,46 & 0,8 & a 17 . & -099 & Qei1 & 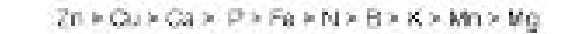 \\
\hline 12 & Anglssob & 0,06 & 0.74 & 0,45 & $-0,17$ & 0,0 . & -034 & 0,80 & 0,02 & 0,00 & $-0,73$ & 0,39 & $\mathrm{a}>\mathrm{Cu}>\mathrm{B}>\mathrm{Ca}>\mathrm{Mg}>\mathrm{N} \cap>\mathrm{N}>\mathrm{K}>\mathrm{Fe}>\mathrm{Zn}$ \\
\hline 16 & angseot & $-11,5 ?$ & 600 & $-10,0$ & $-0,41$ & ars. & $-0 \leqslant 0$ & $0,0 \Omega$ & 025 & 119 & 137 & abo & $K>M_{B}>N>C_{B}>\theta>Z n>P>M n>F_{E}=C u$ \\
\hline 21 & Law: & 0,27 & 0.22 & $-0,78$ & $-0,54$ & $0 \pi$ & 068 & 0,04 & 1.22 & -158 & -0.28 & 0,83 & $F_{u}>K>C a>C u>Z n>P>N>B>M g>V n$ \\
\hline 22 & angessob & 0,10 & 0,01 & $-0,38$ & 0,15 & 0 : & 0,8 & 0,34 & $-0,51$ & $-9,42$ & 028 & 0,34 & $\mathrm{M}>\mathrm{Fe}>\mathrm{K}>\mathrm{Z} n>\mathrm{P}>\mathrm{N}>\mathrm{Ca}>\mathrm{Na}>\mathrm{B}>\mathrm{Cu}$ \\
\hline 210 & angseck & -1.10 & 0,14 & 0,62 & $-0,14$ & $0.2 \%$ & 011 & 0,$4 ;$ & a,ja & 018 & $00 \mathrm{~s}$ & ass & $N>Z n>C A>C,>B>P>F=H B>N n>K$ \\
\hline 25 & W tasale. & $-0,30$ & $\rightarrow \infty 2$ & $-0,40$ & 1.04 & 1,30 & 078 & 0,74 & -247 & 050 & 0.37 & 0.82 & $M_{n}=Z_{n}>K>N>F=C_{u}>F_{e}=B>C a>V_{A}$ \\
\hline $3 t$ & Letosacolo & 0,23 & 021 & 0,20 & $-0,16$ & $-9, c 2$ & $-\square \in a$ & 0,00 & 0,65 & 0 at & 0,98 & $a_{41}$ & $M_{7}>M g>B>C a>Z_{n}>K>N>P>F e>C$ \\
\hline 35 & * & $-0,00$ & 070 & 0,85 & 061 & 0,29 & Q14 & 0,20 & 0,24 & -005 & $-0 \leq 9$ & 014 & $F_{\theta}=Q_{u}=M_{y} \times M_{r}>Z n>N>B=C d>K=P$ \\
\hline 37 & anglanck & 1,22 & $0 a 1$ & $-0,73$ & 1.74 & $-1,15$ & 0.00 & 0,17 & $a c e$ & $-1,01$ & -037 & $a \in$ & $M g=F a>K>C u>Z n>P>M n>Q>N>C Q$ \\
\hline $3 a$ & argissch & 0,63 & $-0,88$ & 9.65 & 0,23 & $1,0 \mathrm{G}$ & 019 & 0,88 & 1,03 & $-1,25$ & -130 & $a 75$ & 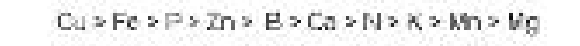 \\
\hline 40 & Navesolo & 000 & 0.15 & $-0,36$ & 0,17 & $-0,51$ & -052 & 0,01 & 0,24 & asa & 060 & $a 32$ & $\mathrm{~B} \times \mathrm{Ng}>\mathrm{K}>\mathrm{P} \times \mathrm{N}>\mathrm{Zn}>\mathrm{Cg}>\mathrm{Mn} \times \mathrm{Fe}_{\mathrm{E}}>\mathrm{O}$ \\
\hline 52 & Latosecio & $-a 0$ & 0,30 & $-0,01$ & 0,42 & 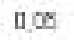 & 071 & 0,50 & 0,02 & .973. & -122 & Q34 & $C_{u} \geqslant P=F_{c}>N>K>V_{n}>M_{a}>C_{a}>Z_{n}>a$ \\
\hline 63 & $*$ & 0,17 & 023 & 0,43 & 0,57 & 0,27 & 052 & $0, \mathrm{ye}$ & 0,40 & 002 & 052 & 0.41 & $\mathrm{Cg}>\mathrm{K}>\mathrm{Zn}>\mathrm{Mg}>\mathrm{P}>\mathrm{N}>\mathrm{Mn}>\mathrm{B}=\mathrm{Cu}>\mathrm{Fe}$ \\
\hline ot & • & $-0.0 t$ & +20 & $a+3$ & $-0,45$ & $-0,12$ & 0.10 & 0,50 & 0,0 & 027 & -018 & 028 & $N_{0}=\mathrm{Ca}>\mathrm{P}>\mathrm{O}>\mathrm{N}>\mathrm{Mh}>\mathrm{B}>\mathrm{Fe}>\mathrm{K}>\mathrm{Zn}$ \\
\hline 65 & + & a os & 0,9 & 0.25 & $-0,62$ & $a x$. & -200 & 0.84 & $.0,43$ & $\mathrm{a} 7 \mathrm{~s}$ & 022 & a61 & 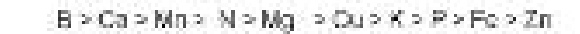 \\
\hline
\end{tabular}

- Eolo råo ctaresificaco

Tabela 3.- Ordens de Solo, Índices de Balanço Nutricional (IBNm), Índices DRIS e Ordem de Limitação de Nutrientes obtidas a partir de teores foliares de nutrientes em pomares de mangueira Tommy Atkins de alta produtividade ( $\geq 250 \mathrm{~kg} / \mathrm{planta})$ no Submédio São Francisco

\begin{tabular}{|c|c|c|c|c|c|c|c|}
\hline$x$ & $Y$ & EQUACAAO & $r$ & $x$ & $Y$ & EQUACAO & $\mathrm{I}$ \\
\hline $\mathrm{Ng} \times \mathrm{g}$ & ID N & $0053 \mid 0^{i} i x-5$ & 0,57 & B $\mathrm{mg} \mathrm{kg}$ & IDE & $5857 \operatorname{Ln}\{x\}-1$ & 0,88 \\
\hline P & IDP & 14 & 0,81 & $Z_{71}$ & $10 Z_{7}$ & 112 & 0.89 \\
\hline $\begin{array}{l}\mathrm{Kg} \mathrm{kg}^{\prime} \\
\mathrm{Cg} g \mathrm{~kg}^{-1}\end{array}$ & $\begin{array}{l}10 \mathrm{~K} \\
1 \mathrm{DCz}\end{array}$ & $\begin{array}{l}Y=0,23 \\
Y=0,07\end{array}$ & $\begin{array}{l}0.68 \\
0.74\end{array}$ & $\begin{array}{l}\text { Mn mg kg } \\
\text { Fe mg kg }\end{array}$ & $\begin{array}{l}10 \mathrm{Mn} \\
10 \mathrm{Fe}\end{array}$ & $\begin{array}{r}.3199 \\
.9975\end{array}$ & $\begin{array}{l}0,89 \\
0,85\end{array}$ \\
\hline$M g g \mathrm{~kg}^{\prime}$ & $\mathrm{ID} \mathrm{Mg}$ & $Y=1,7114 \mathrm{Lm}(x)-1,6016$ & c. 73 & Cu $m g \mathrm{~kg}$ & $10 \mathrm{Cu}$ & $Y=1.59 / \mathrm{Ln}\{x\}-5.0426$ & 0,92 \\
\hline
\end{tabular}

Tabela 4.- Equações e coeficientes de correlação entre índices DRIS (ID) e teores foliares (x) de macro e de micronutrientes em folhas de mangueira Tommy Atkins no Submédio São Francisco

\begin{tabular}{|c|c|c|}
\hline SUBPOPULACุÁ & LIMITAÇÁO POR DEFICIÉNCIA & ORDEM DE LNITAÇÁO \\
\hline \multirow{4}{*}{$\begin{array}{l}\text { ALTA } \\
\text { PRCDUT }\end{array}$} & + LIMTANTE & $\begin{array}{c}\mathrm{Mn}>\mathrm{Cs}=\mathrm{Mg}=\mathrm{B}=\mathrm{Cu}=\mathrm{Fe}=\mathrm{Zn}>\mathrm{N}=\mathrm{K}>\mathrm{P} \\
17,6511,76=11,76 \quad 11,76=11,76=11,76=11,785,885880\end{array}$ \\
\hline & $2^{\circ}+\underset{\%}{\operatorname{LIMTANTE}}$ & 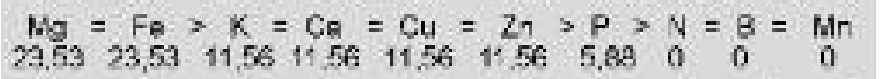 \\
\hline & $3^{\circ}+\underset{\%}{\text { LIMTANTE }}$ & 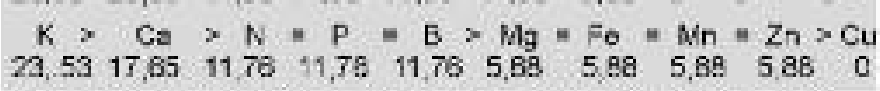 \\
\hline & Bequiência Média & 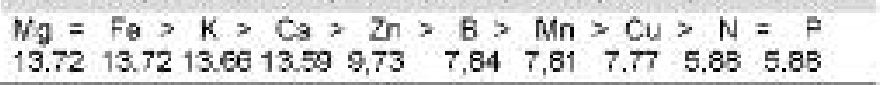 \\
\hline \multirow{4}{*}{$\begin{array}{l}\text { BAXA } \\
\text { PRODUTYDADE }\end{array}$} & $+\underset{\%}{+ \text { LIMTANTE }}$ & 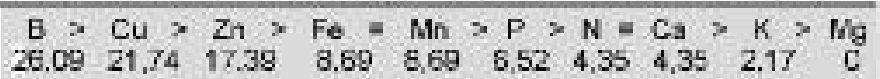 \\
\hline & $2^{\circ}+\underset{\%}{\text { LIMTTANTE }}$ & 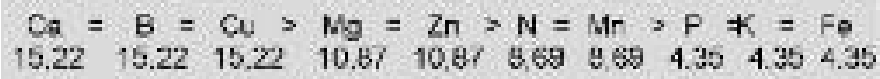 \\
\hline & $3^{\circ}+\underset{\mathscr{K}}{\operatorname{LIMTANTE}}$ & 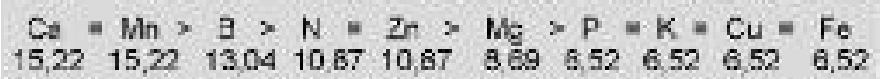 \\
\hline & SEQÜÉNCIA MECAA & 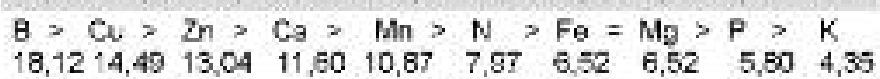 \\
\hline
\end{tabular}

Tabela 5.- Resumo da ordem de limitação da produtividade por nutrientes em deficiência e respectivas porcentagens de ocorrência nos pomares de mangueira Tommy Atkins de alta e de baixa produtividade 
O P limitou as safras por excesso, em cerca de $15,22 \%$ dos pomares de baixa produtividade. Os teores foliares de $\mathrm{P}$ variaram nos pomares de baixa produtividade de 0,5 a 2,9 $\mathrm{g} / \mathrm{kg}$, com média igual a $1,4 \mathrm{~g} / \mathrm{kg}$, enquanto que nos pomares de alta produtividade esses teores variaram de 0,76 a 1,60 , com média igual a $1,16 \mathrm{~g} / \mathrm{kg}$. Nos pomares de baixa produtividade encontrou-se uma correlação negativa entre os teores foliares de $\mathrm{P}$ e a produtividade das árvores $\left(-0,46^{* *}\right)$, sugerindo que teores muito altos do elemento possam estar causando desequilíbrios nutricionais. Igual situação foi verificada nos pomares de alta produtividade $(r$ $\left.=-0,56^{*}\right)$. Tal constatação é coerente com o fato de que o $P$ é o quinto elemento mais exportado pela mangueira, após K, N, Ca e Mg (Quaggio, 1996).

O coeficientes de correlação entre o IBN e os índices DRIS para o $\mathrm{P}$ nos pomares de baixa produtividade foi da ordem de $0,39{ }^{* *}$ (significativo pelo teste $t$ ) sendo os dados descritos pela equação $y=7,5882+1,9368 x$. Na medida em que os teores foliares de $\mathrm{P}$ aumentaram nesses pomares, também aumentaram os valores de IBN, conseqüentemente, aumentando os desequilíbrios nutricionais nas árvores.

Em 41,13 \% dos pomares de alta produtividade os macronutrientes são os mais limitantes por excesso, destacando-se $\mathrm{K}$ e $\mathrm{Mg}$, enquanto que em 58,81\% dos pomares os micronutrientes foram os mais limitantes por deficiência, destacando-se Mn, B, Cu e Fe (Tabela 6).

Em 39,86 \% dos pomares de baixa produtividade os macronutrientes são os mais limitantes por excesso, destacando-se $\mathrm{P}$ e $\mathrm{K}$, enquanto que em 58,69 \% dos pomares os micronutrientes foram os mais limitantes por deficiência, destacando-se Fe, Cu e Zn (Tabela 6).

\begin{tabular}{|c|c|c|}
\hline POPULAÇĀO & $\begin{array}{l}\text { NUTRIENTE EM } \\
\text { EXCESSO }\end{array}$ & ORDEM DE UIMITAÇĀO \\
\hline \multirow{3}{*}{$\begin{array}{l}\text { ALTA } \\
\text { FRODUTIVIDADE }\end{array}$} & $+\underset{\%_{0}}{+ \text { LIMTANTE }}$ & 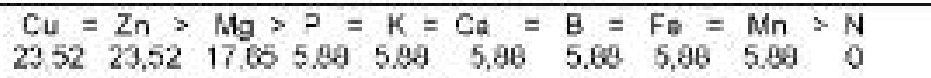 \\
\hline & $\underset{\%}{Z^{a}}+$ LIRITANTE & 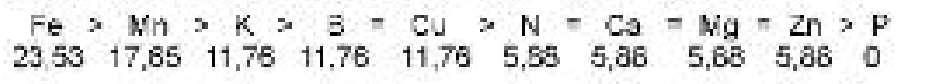 \\
\hline & $3^{\circ}+\underset{\%_{0}}{\operatorname{LIBIT}} / N N_{T}$ & 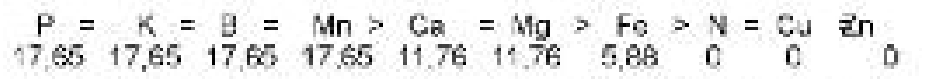 \\
\hline \multirow{5}{*}{$\begin{array}{l}\text { EAIXA } \\
\text { PRODUTIVIDADE }\end{array}$} & Seqūonncis Mádia & 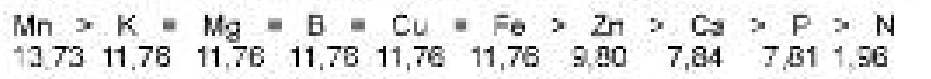 \\
\hline & $+\underset{\& \infty}{\text { LIMTANTE }}$ & 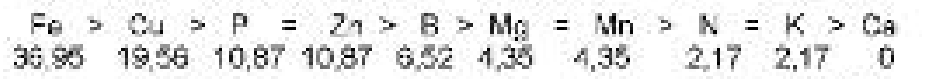 \\
\hline & $\underset{\%}{2^{n}+\operatorname{LIMITANTE}}$ & 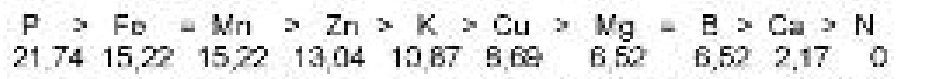 \\
\hline & $3^{\circ}+\underset{\%}{\text { LISITANTE }}$ & 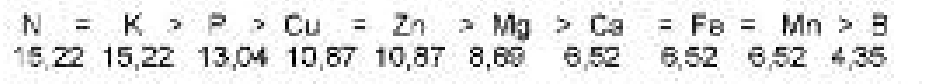 \\
\hline & Sequảncis Média & 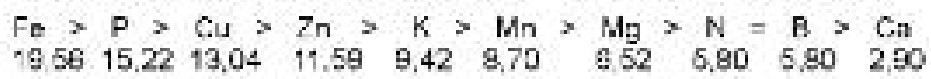 \\
\hline
\end{tabular}

Tabela 6.- Resumo da ordem de limitação da produtividade por nutrientes em excesso e respectivas porcentagens de ocorrência nos pomares de mangueira Tommy Atkins de alta e de baixa produtividade

As concentrações foliares mais altas de $\mathrm{P}$, da ordem de 2,20 a $2,90 \mathrm{~g} / \mathrm{kg}$ (70\% a $148 \%$ superiores ao valor da norma foliar para este elemento, respectivamente) foram todas constatadas em pomares de baixa produtividade e em solos com altas concentrações de $P$ disponível (Mehlich 1) nas camadas de $0-20 \mathrm{~cm}$ e,ou, de $20-40 \mathrm{~cm}$, notadamente na parte mais superficial, onde as concentrações se situaram de 56 a $155 \mathrm{mg} / \mathrm{dm}^{3}$, interpretados na região como altas (Cavalcanti, 1998).

Stassen et al. (1997) trabalhando com mangueiras 'Sensation', enxertadas sobre 'Sabre", observaram que as mesmas, quando com a idade de seis anos, apresentavam na matéria seca das folhas $29,6 \%$ do fósforo contido na planta, o restante estando contido nas raízes $(17,9 \%)$, nos ramos novos $(16,6 \%)$, nos frutos $(14,9 \%)$, no lenho $(11,7 \%)$ e na casca $(9,3 \%)$. Esses dados sugerem que, embora as folhas constituam o compartimento da mangueira que proporcionalmente contenha a maior porcentagem do $\mathrm{P}$ da planta, parcela significativa desse nutriente $(70,4 \%)$, está contida no conjunto dos demais órgãos. Logo, os altos teores do nutriente na folha constatados em alguns pomares, embora possam denotar alta disponibilidade do nutriente no solo, não expressam a totalidade dessa disponibilidade.

Pode estar havendo excesso na aplicação de K em 9,42 \% dos pomares de baixa produtividade, bem como em 11,76 $\%$ dos pomares de alta produtividade.

Os nutrientes $\mathrm{Mn}, \mathrm{K}, \mathrm{Mg}, \mathrm{B}, \mathrm{Cu}$, e Fe podem estar sendo aplicados em excesso, quando se adicionam calcário, fertilizantes contendo $\mathrm{K}$ e micronutrientes ou em aplicações fitossanitárias envolvendo a presença do $\mathrm{Mn}$, ou quando não complementados proporcionalmente pelos demais nutrientes, nos pomares de alta produtividade (Tabela 6). Os Índices de Balanço Nutricional (IBN), resultantes da soma dos valores absolutos dos índices DRIS encontrados para cada nutriente considerado, indicam que quanto menores forem, melhor será o estado nutricional das plantas 
(Sumner, 1977). Neste trabalho, os Índices de Balanço Nutricional médio $\left(\mathrm{IBN}_{\mathrm{m}}\right)$ nos pomares de baixa produtividade variaram de 0,30 a 3,72 , com média igual a 0,80 , enquanto que nos pomares de alta produtividade variaram de 0,26 a 0,80 , média igual a 0,50 , sendo, portanto, valores mais baixos nesse grupo de pomares, podendo indicar melhor balanço nutricional. Entretanto, encontram-se valores de $\mathrm{IBN}_{\mathrm{m}}$ menores que 0,50 entre os pomares de baixa produtividade. Verificando as informações registradas nesses pomares, percebe-se que na maioria dos mesmos a produtividade foi superior a 150 $\mathrm{kg} /$ planta, enquanto nos demais a produtividade foi bem menor que esse patamar. Supondo-se que uma "planta se encontre em estado nutricional adequado, seria de se esperar que a mesma apresentasse uma produtividade, pelo menos correspondente àquele estado nutricional" (Prévot e Ollagnier, 1957, citados por Leite, 1993). Em trabalhos desenvolvidos por Leite (1993) e por Costa (1995) foram encontrados valores altos de IBN em lavouras de alta produtividade, aparentemente contrariando um dos fundamentos básicos do DRIS, que consiste no fato de que o "status" nutricional da planta, ou seja, seu equilíbrio relativo entre os nutrientes assimilados integra, dentro de certos limites, as condições capazes de refletirem o seu potencial produtivo (Beaufils, 1973). Conforme Costa (1995), em lavouras de baixa produtividade o IBN pode ser alto ou baixo, dependendo de o fator limitante na produtividade estar ou não relacionado ao equilíbrio nutricional. Nas lavouras de baixa produtividade que apresentaram altos valores de IBN, o mais provável é que a causa para a baixa produtividade esteja relacionada ao desbalanço nutricional.

Leite (1993) informa que no caso de lavouras de baixa produtividade, apresentando IBN até mesmo inferior aos da população de referência, tal fato indica que as mesmas podem estar até em situação melhor em termos de equilíbrio nutricional, do que aquelas de alta produtividade, sugerindo que a principal limitação na produtividade para essas lavouras tenha sido de ordem não nutricional. Isso está de acordo com o que afirmou Snoeck (1984), citado por Leite (1993), de que nem sempre uma planta em equilíbrio nutricional adequado, significa que a mesma terá alta produtividade, sendo apenas o inverso verdadeiro, ou seja, a alta produtividade das culturas só é alcançada quando as plantas estiverem em equilíbrio nutricional ótimo. Em situações onde o equilíbrio nutricional estava dentro de limites adequados e a planta apresentou baixa produtividade, a limitação foi de ordem não nutricional, podendo ser tanto ambiental (Chapin, 1984, citado por Leite, 1993), como também fisiológica.

Para a interpretação dos índices DRIS incorporou-se o método do Potencial de Resposta à Adubação (PRA) (Wadt, 1996). Para tanto, nos pomares de baixa e de alta produtividade, os índices DRIS para cada nutriente (Tabelas 2 e 3) foram classificados segundo o potencial de resposta à adubação, em respostas: positiva $(p)$, positiva ou nula $(\mathrm{pz})$, nula $(\mathrm{z})$, negativa ou nula $(\mathrm{nz})$ e negativa $(\mathrm{n})$ (Tabelas 7 e 8$)$.

\begin{tabular}{|c|c|c|c|c|c|c|c|c|c|c|}
\hline \multirow[b]{2}{*}{ Pomar } & \multicolumn{10}{|c|}{ Potenclal de resposta à adubą̧ão com cada nutriente } \\
\hline & $\mathrm{N}$ & $\mathbf{P}$ & $\mathbf{K}$ & $\mathrm{Ca}$ & Mg & B & Cu & $\mathrm{Fe}$ & fin & $\mathrm{Zn}$ \\
\hline 1 & $p z$ & $\mathrm{nz}$ & $z$ & $z$ & $z$ & $\rho$ & $z$ & $n$ & $z$ & $z$ \\
\hline 2 & $z$ & $n z$ & $z$ & $z$ & $p z$ & $p$ & $z$ & n & $z$ & $z$ \\
\hline 3 & $p$ & $z$ & $z$ & $p z$ & $z$ & $p z$ & $z$ & $n$ & $z$ & $z$ \\
\hline 4 & $z$ & $n$ & $z$ & $z$ & $z$ & $\mathrm{p}$ & $z$ & $\mathrm{nz}$ & $x$ & $z$ \\
\hline 5 & $z$ & $z$ & $z$ & $z$ & $z$ & $z$ & n & $\mathrm{nz}$ & $z$ & $P$ \\
\hline B & nz & $z$ & $z$ & $z$ & $\boldsymbol{z}$ & $\mathrm{pz}$ & p & n & $z$ & $z$ \\
\hline 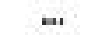 & & & & & & & & & & \\
\hline 57 & $z$ & $z$ & $z$ & $z$ & $z$ & $z$ & $n$ & $p Z$ & $z$ & D \\
\hline 58 & $\bar{n}$ & $\bar{z}$ & $z$ & $z$ & $\mathrm{z}$ & $z$ & nz & $z$ & $\bar{z}$ & p \\
\hline 60 & 2 & $N$ & $z$ & 2 & 2 & 2 & 2 & $z$ & 2 & $p$ \\
\hline 62 & $z$ & $\mathrm{~N}$ & $\mathrm{n} 2$ & $z$ & $z$ & P & $z$ & $z$ & $\mathrm{pZ}$ & nz \\
\hline 63 & $z$ & $z$ & $z$ & $z$ & $z$ & $p$ & $\mathrm{pz}$ & $z$ & $\mathrm{pz}$ & $n$ \\
\hline 64 & $z$ & $\bar{z}$ & $\mathrm{nz}$ & 2 & $z$ & $p$ & $z$ & $\pi$ & $x$ & 2 \\
\hline 66 & $z$ & $z$ & $z$ & $p 2$ & $z$ & P & $z$ & $z$ & $z$ & $n$ \\
\hline 67 & $\bar{z}$ & $Z$ & $z$ & $p$ & $n z$ & $\mathrm{pz}$ & $z$ & $n$ & $z$ & $z$ \\
\hline 68 & $z$ & $\angle$ & $\mathrm{pz}$ & $z$ & $z$ & 0 & $\mathrm{nz}$ & $z$ & $z$ & $\mathrm{n}$ \\
\hline
\end{tabular}

pasitiva (p), posisiva ou nula (pz), negativa (n); regativa ou rula (rmz) e nula zl

\begin{tabular}{|c|c|c|c|c|c|c|c|c|c|c|}
\hline \multirow[b]{2}{*}{ Pomar } & \multicolumn{10}{|c|}{ Potencial de resposta à adubaģảo com cada nutriente } \\
\hline & $\mathbf{N}$ & $P$ & K & $\mathrm{Ca}$ & Fig & B & $\mathrm{Cu}$ & Fe & Min & $\mathrm{Zn}$ \\
\hline 10 & $z$ & 2 & $z$ & p & $p 2$ & $m z$ & $z$ & 2 & $z$ & n \\
\hline 12 & $z$ & 2 & $n z$ & $z$ & $n$ & $z$ & pz & 2 & re & p \\
\hline 13 & $z$ & $\beta$ & $n z$ & 2 & $z$ & $z$ & $\mathrm{pz}$ & $n e$ & $z$ & n \\
\hline$=$ & & & & & & & & & & \\
\hline 52 & $z$ & $z$ & 2 & $n z$ & $z$ & $n$ & $p$ & 2 & $z$ & $\mathrm{nz}$ \\
\hline 59 & $z$ & 2 & $\rho z$ & p & $z$ & $\mathrm{nz}$ & $r 2$ & $\mathrm{r}$ & $z$ & $z$ \\
\hline 61 & $z$ & $\mathrm{pz}$ & $n z$ & $\mathrm{pz}$ & p & $z$ & $z$ & $n$ & $z$ & $n$ \\
\hline 65 & $z$ & 2 & 2 & $p z$ & 2 & $\mathrm{P}$ & $z$ & ru & $z$ & $\mathrm{n}$ \\
\hline
\end{tabular}

fositva (p); positiva cu nula (pz), negativa $\{n\rangle$; negativa cu nula $\langle(n z)$ e nula z)
Tabela 7.- Potencial de resposta à adubação com macro e micronutrientes em pomares de mangueira Tommy Atkins de baixa produtividade

Tabela 8.- Potencial de resposta à adubação com macro e micronutrientes em pomares de mangueira Tommy Atkins de alta produtividade 
O potencial de resposta à adubação com macronutrientes mostrou-se positivo em $41,16 \%$ dos pomares de alta produtividade, enquanto que apenas em 15,22 \% dos de baixa produtividade, talvez devido à maior exportação de nutrientes nas colheitas no primeiro caso, ou em decorrência da maior aplicação desses nutrientes em detrimento da aplicação de micronutrientes nos pomares de baixa produtividade.

Quanto aos micronutrientes, o potencial de resposta à adubação foi positivo em $84,77 \%$ dos pomares de baixa produtividade, ao passo que em $58,81 \%$ dos de alta produtividade, nos quais, como se supõe, a despeito da aplicação desses nutrientes, isso não tem sido suficiente, motivo pelo que adubações criteriosas com esses elementos devem ser realizadas.

A limitação por deficiência de nutrientes pelo método do Potencial de Resposta à Adubação foi: $\mathrm{Mg}>\mathrm{Cu}=\mathrm{K}=\mathrm{Fe}>$ $\mathrm{Ca}=\mathrm{B}>\mathrm{Mn}=\mathrm{Zn}=\mathrm{N}=\mathrm{P}$, e $\mathrm{B}>\mathrm{Cu}=\mathrm{Zn}>\mathrm{Ca}>\mathrm{N}>\mathrm{Fe}>$ $\mathrm{Mn}>\mathrm{P}>\mathrm{K}=\mathrm{Mg}$, nos pomares de alta e de baixa produtividade, respectivamente. Dessa forma, por esse método, tem-se a informação de que nutrientes devem ser prioritariamente aplicados nas adubações.

No que diz respeito à limitação da produtividade por excesso de nutrientes, a ordem de limitação foi: $\mathrm{Fe}>\mathrm{K}=\mathrm{Mg}$ $=\mathrm{Cu}=\mathrm{Zn}>\mathrm{Ca}=\mathrm{B}>\mathrm{Mn}>\mathrm{N}=\mathrm{P}, \mathrm{e}, \mathrm{Fe}>\mathrm{P}>\mathrm{Cu}>\mathrm{Zn}>\mathrm{Mn}$ $=\mathrm{K}>\mathrm{B}>\mathrm{Mg}>\mathrm{N}>\mathrm{Ca}$, nos pomares de alta e de baixa produtividade, respectivamente.

Pelo método do Potencial de Resposta à Adubação (PRA) o $\mathrm{P}$, depois do $\mathrm{Fe}$, foi classificado como o nutriente que mais limitou, por excesso, a produtividade nos pomares de baixa produtividade (Tabela 9).

\section{Conclusões}

Os maiores valores de Índices de Balanço Nutricional médio $\left(\mathrm{IBN}_{\mathrm{m}}\right)$ verificados para os pomares de baixa produtividade demonstraram o potencial deste índice na avaliação do estado nutricional de mangueiras, bem como a necessidade de adubações mais criteriosas para tais pomares.

A utilização do conceito de potencial de resposta à adubação na interpretação dos índices DRIS mostrou-se uma ferramenta eficaz, principalmente nos pomares de baixa produtividade, onde os desequilíbrios nutricionais foram maiores, havendo bem maior confirmação da seqüência de deficiência a excessos, auxiliando o diagnóstico final, indicando os nutrientes que realmente estão em deficiência ou em excesso.

A limitação da produtividade de manga Tommy Atkins por excesso de nutrientes, pelo método do Potencial de Resposta à Adubação obedeceu à seguinte ordem: $\mathrm{Fe}>\mathrm{K}$ $=\mathrm{Mg}=\mathrm{Cu}=\mathrm{Zn}>\mathrm{Ca}=\mathrm{B}>\mathrm{Mn}>\mathrm{N}=\mathrm{P}, \mathrm{e}, \mathrm{Fe}>\mathrm{P}>\mathrm{Cu}>\mathrm{Zn}$ $>\mathrm{Mn}=\mathrm{K}>\mathrm{B}>\mathrm{Mg}>\mathrm{N}>\mathrm{Ca}$, nos pomares de alta e de baixa produtividade, respectivamente.

A limitação da produtividade por deficiência de nutrientes foi: $\mathrm{Mg}>\mathrm{Cu}=\mathrm{K}=\mathrm{Fe}>\mathrm{Ca}=\mathrm{B}>\mathrm{Mn}=\mathrm{Zn}=\mathrm{N}=\mathrm{P}$, e $\mathrm{B}>\mathrm{Cu}$ $=\mathrm{Zn}>\mathrm{Ca}>\mathrm{N}>\mathrm{Fe}>\mathrm{Mn}>\mathrm{P}>\mathrm{K}=\mathrm{Mg}$, nos pomares de alta e de baixa produtividade, respectivamente.

\begin{tabular}{|c|c|c|c|c|c|c|c|}
\hline \multirow[b]{2}{*}{ Nutriente } & \multicolumn{3}{|c|}{ Alta produttvidade } & \multicolumn{4}{|c|}{ Babxa produtbidade } \\
\hline & LF & NL & LE & Nutriente & $L=$ & NL & LE \\
\hline $\mathrm{N}$ & 11,76 & 92,36 & 5,99 & $\mathrm{~N}$ & 17,39 & 76,09 & 6,52 \\
\hline $\mathrm{F}$ & 11.76 & 02.35 & 5.88 & $\mathrm{p}$ & 6.70 & 56.52 & 3478 \\
\hline K & 29,41 & 41,12 & 29,41 & K & 6,52 & 78,26 & 1521 \\
\hline $\mathrm{Ca}$ & 23.52 & $52 \$ 4$ & 23.53 & Ca & 23,91 & 73.91 & 2,17 \\
\hline $\mathrm{Na}_{\mathrm{g}}$ & 36,29 & 35,29 & 29,41 & $\mathrm{Mg}$ & 6,52 & 84,78 & 8,70 \\
\hline $\mathrm{B}^{2 \pi}$ & 23,52 & 52,84 & 23,53 & $B$ & 39,13 & 50,00 & 1087 \\
\hline $\mathrm{Cu}$ & 28.41 & 41.18 & 29.41 & $\mathrm{Cu}$ & 28,26 & 43.48 & 2825 \\
\hline Fo & 28,41 & 29.41 & 41,17 & Fo & 15,21 & 39,13 & 45.65 \\
\hline Mrr & 17,65 & 6470 & $17 B A$ & Mrı & 13,04 & 71,74 & 1522 \\
\hline $\mathrm{Zn}$ & 17,64 & 6284 & 29,41 & Zn & 28,25 & 62,17 & 1956 \\
\hline
\end{tabular}

Tabela 9.- Porcentual de pomares de mangueira Tommy Atkins de alta e de baixa produtividade em que cada nutriente limita a produti- vidade pelo Potencial de Resposta à Adubação

\section{Bibliografia}

Alvarez V., V. H.; Leite, R. de A. (1999). Fundamentos estatísticos das fórmulas usadas para o cálculo dos índices DRIS. Boletim Informativo da Sociedade Brasileira de Ciência do Solo. 24, 1: 13-15.

Baldock, J. O.; E. E. Schulte (1996). Plant analysis with standardized scores combines DRIS and sufficiency range approaches for corn. Agronomy Journal. 88: 448-456.
Beaufils, E.R. (1973). Diagnosis and recommendation integrated system (DRIS). A general scheme of experimentation based on principles developed from research in plant nutrition. Soil Sci. Bull, 1, University of Natal, Pietermaritzburg, South Africa.

Bergmann, W. (1992). Nutritional disorders of plants; development, visual and analytical Diagnosis. New York, Gustave Fischer Verlug Gena. Stuttgart.

Camargo, P. N. de; Silva, O. (1990). Manual de adubação foliar. S. Paulo, HERBA, 256 p. 
Cavalcanti, F. J. de A. (1998). Recomendações de adubação para o Estado de Pernambuco (2a aprox.) 2 ed. rev. Recife: IPA.

Costa, A. N. (1995). da. Uso do sistema integrado de diagnose e recomendação (DRIS), na avaliação do estado nutricional do mamoeiro (Carica papaya L.) no Estado do Espírito Santo. Viçosa, UFV. (Tese D.Sc.)

Horst, W. J. (1998). The physiology of manganese toxicity. In: GRAHAM, R. D.; HANNAM, R. J.; UREN, N. C. eds. Manganese in soil and plants, Kluwer Academic. p.175-188.

Jones, C. A. (1981). Proposed modifications of the diagnosis and recommendation integrated system (DRIS) for interpreting plant analysis. Commun. Soil Sci. and Plant Anal. 12: 785-974.

Leite, R. A. (1993). Avaliação do estado nutricional do cafeeiro conilon no Estado do Espírito Santo utilizando diferentes métodos de interpretação de análise foliar. Viçosa. Universidade Federal de Viçosa. (tese D. Sc).

Pinto, P. A. da C. (2002). Avaliação do estado nutricional da mangueira Tommy Atkins pelo DRIS e da qualidade pós colheita de frutos no Submédio São Francisco. Viçosa: UFV. (Tese de doutorado). Disponível em: http://www.augustocoimbra.xpg.com.br/tese.zip; [12 julho 2007]

Quaggio, J. A. (1996). Adubação e calagem para a mangueira e qualidade dos frutos. In: São José, A. R.; Souza, I. V. B.; Filho, J. M. ; Morais, O. M. Manga, tecnologia de produção e mercado. Vitória da Conquista, BA, DFZ/UESB. p.106-135.

Reis JR., R. dos A. (1999). Diagnose nutricional da cana de - açúcar com uso do sistema integrado de diagnose e recomendação (DRIS). Campo dos Goytacazes, RJ. (Tese D.Sc.)
Samra, J. S.; Arora, Y. K. (1997). Mineral nutrition. In: Litz, R. E. The mango: botany, production and uses. New York, CAB International.

Schaffer, B.; Larson, K. D.; Snyder, G. H.; Sanchez, C. A. (1988). Identification of mineral deficiences associated with mango decline by DRIS. Hortscience. 23, 3: 617-6198.

Snyder, G. H.; Kretschmer, A. E. (1988). A DRIS analysis for bahiagrass pastures. Soil and Crop Science Society of Florida Proceedings. 47: 56-59.

Stassen, P. J. C.; Terblanche, J. H.; Strydom, D. K. (1981). The effect of time and rate of nitrogen application on development and composition of peach trees. Agroplantae. 13: $55-61$.

Stassen, P. J. C.; Vuuren, B. H. P. J. Van; Davie, S. J. (1997). Macro elements in mango trees: uptake and distribution. $\mathrm{S}$. A. Mango Grower's Association Yearbook. 17, p.16.

Sumner, M. E. (1977). Effect of corn leaf sampled on N, P, $\mathrm{K}, \mathrm{Ca}$ and $\mathrm{Mg}$ content and calculated DRIS indices. Communication In Soil Science and Plant analysis, v. 8, p.269-280.

Wadt, P. G. S. (1995). Software para cálculo de índices DRIS, índices IBN mediante diferentes fórmulas. UFV, Viçosa, MG. (dois disquetes 3,5")

Wadt, P. G. S. (1996). Os métodos da chance matemática e do Sistema Integrado de Diagnose e Recomendação (DRIS) na avaliação nutricional de plantios de eucalipto. Viçosa, UFV. (tese D.Sc.) 